\title{
Layer Spacings in Coherently Strained Epitaxial Metal Films
}

\author{
W. F. Chung, ${ }^{1}$ Y. J. Feng, ${ }^{1}$ H. C. Poon,${ }^{2}$ C. T. Chan, ${ }^{1}$ S. Y. Tong, ${ }^{3}$ and M. S. Altman ${ }^{1, *}$ \\ ${ }^{1}$ Department of Physics, Hong Kong University of Science and Technology, Hong Kong, China \\ ${ }^{2}$ Department of Physics, The University of Hong Kong, Hong Kong, China \\ ${ }^{3}$ Department of Physics and Materials Science, City University of Hong Kong, Hong Kong, China
}

(Received 30 January 2003; published 27 May 2003)

\begin{abstract}
Laterally resolved measurements of the quantum size effect (QSE) in electron reflectivity are made with low energy electron microscopy on coherently strained Ag films on a W(110) surface. The evolution of the total film thickness with increasing number of atomic layers is determined accurately by dynamical theory analysis of the QSE features. Combined with a model of layer spacings obtained from first-principles calculations, this provides for a novel approach to determine the buried interface layer spacing, which is inaccessible to other methods.
\end{abstract}

PACS numbers: 68.55.Jk, 68.35.Ct, 68.37.Nq, 81.40.Jj

Ultrathin films are known to be strongly influenced by their interaction with the supporting substrate. Films are often strained due to lattice mismatch with the substrate. In the coherently strained regime, prior to introduction of misfit dislocations, lateral tensile and compressive strains couple to out-of-plane film strain in such a way that roughly conserves the atomic volume. This phenomenon is well understood by elasticity theory [1]. However, the interface layer spacing and modification of surface relaxations by lateral film strain are not so well understood. In the ultrathin regime, where quantization effects are most apparent, the surface and interface regions can be substantial components of the film and are therefore essential to models of film structure.

Several techniques exist for studying surface structure. Most are not useful for probing film structure from the surface to the buried interface. In x-ray reflectivity, interference fringes generated by reflection at the surface and interface discontinuities have been used successfully for determining film thickness and roughness [2]. In this Letter, we exploit an analogous phenomenon for electrons, termed the quantum size effect (QSE) in electron reflectivity [3-6], to determine the total film thickness. Numerous QSEs involving electrons near the Fermi level are known to occur in thin films as a result of the confinement by the surface and interface with the substrate [7-9]. These QSEs have clearly shown a dependence on the number of atomic layers in the film. It is therefore reasonable to expect that QSEs are also very sensitive to the precise distance between surface and interface and possibly other structural details. Although the sensitivity of the QSE in photoemission to strain-induced changes in structure was recognized [9], the QSE is not developed as a tool for studying structure. A challenge to laterally averaging techniques is posed by the coexistence of regions of different film thicknesses in the macroscopic area being probed. In our work, laterally resolved measurements of the QSE in a low energy electron microscope (LEEM) discriminate information from regions of a film that have different numbers of atomic layers. This facilitates the comparison of experimental data to model calculations for idealized uniformly thick films. The evolution of the total film thickness with increasing number of layers in single atomic layer increments can then be determined accurately by dynamical theory analysis of experimental QSE features. Comparison of these experimental results to a model of layer spacings obtained from first-principles calculations is the basis of a novel approach to determine the strained layer spacing in the film and buried interface spacing.

The system under investigation is Ag films on the $\mathrm{W}(110)$ surface. It is known from earlier studies [10,11] that a distorted $\operatorname{Ag}(111)$ film structure is well developed at 2 monolayer (ML) thickness. According to LEED [10], fcc $[1 \overline{1} 0]$ rows of atoms in the distorted $\mathrm{Ag}(111)$ structure are aligned and matched with the substrate [1111] rows [Fig. 1(a)]. Along these rows, the Ag atoms have periodicity that is incommensurate with the W atom periodicity in the matching substrate rows. Row matching is achieved in the film [011] direction by a combination of uniaxial tensile strain and shear strain [Fig. 1(b)]. A distorted $\mathrm{Ag}(111)$ structure was also determined by helium atom scattering [11]. No significant change of in-plane film structure was detected for thickness above $2 \mathrm{ML}$ in the previous $[10,11]$ or the present work. Ag was deposited in our experiments at a rate of $0.33 \mathrm{ML} / \mathrm{min}$ onto a sample held at about $500 \mathrm{~K}$. Measurements were carried out at room temperature. The imaging principle and contrast mechanisms of LEEM have been discussed previously $[5,6]$.

The QSE can be understood to be an interference phenomenon between the electron waves that are reflected from the surface of the film and from the interface between the film and substrate [3-6]. Consequently, the QSE periodically modulates the reflected intensity as a function of both film thickness and energy. This gives rise to prominent quantum interference peaks (QIPs) which, in the kinematical description, have maxima when the 
$\mathbf{a}$
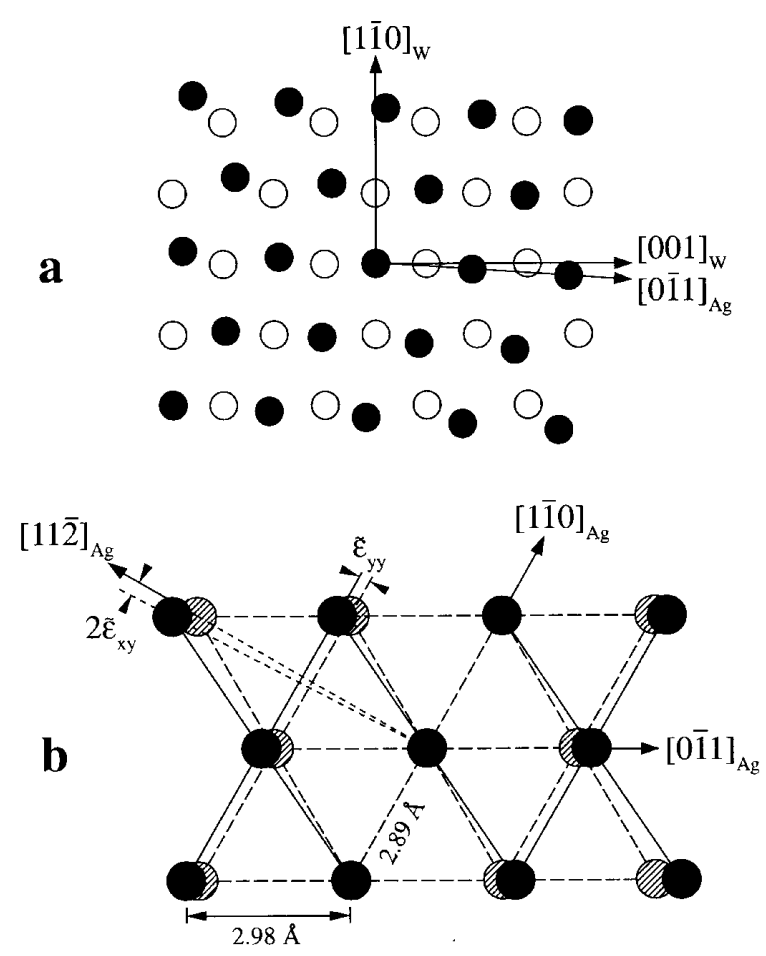

FIG. 1. (a) Structure model for $\mathrm{Ag} / \mathrm{W}(110)$ from Ref. [10] (Ag are solid and $\mathrm{W}$ are open circles). (b) Generation of the structure in (a) by in-plane strain, $\tilde{\varepsilon}_{y y}=0.0313$, and shear strain, $\tilde{\varepsilon}_{x y}=-8.72 \times 10^{-3} \mathrm{rad}$, of the bulk lattice (shaded circles). The substrate and film directions are distinguished by the $\mathrm{W}$ and Ag subscripts, respectively.

in-phase condition $\left(E+V_{0}\right)=\pi^{2} n^{2} / 2 D^{2}$ is met, where $n$ is an integer and $D$ is the total film thickness. Here, $E$ (electron energy above vacuum) and $V_{0}$ (inner potential of film) are in hartrees. It is easy to show that for an $N$-layer thick film, there are $(N-1)$ QIPs between Bragg peaks. In principle, QIPs exist between any two consecutive Bragg peaks at all energies. However, QIPs become very weak at energies above the plasmon threshold, where the electron mean free path in the film is short [12] and the reflectivity from the film-substrate interface is much weaker than reflectivities from the top few layers of the film. This is why QIPs have never been reported in conventional LEED $I(V)$ analysis, which focuses on energies above $30 \mathrm{eV}$, and why it is crucial to work in the very low energy range to study the QSE. A related interference effect occurs between electrons that are reflected from terraces separate by single and multiatom high steps. This interference survives to higher energies because both reflecting surfaces are exposed and absorption is absent. LEED spot profile measurements of this effect have been used to determine island heights in terms of the number of atomic layers [13].

The QSE is manifested vividly in LEEM as quantum size contrast between regions of different film thickness (Fig. 2). By varying the incident electron energy and measuring the intensity in uniformly thick regions, aver-

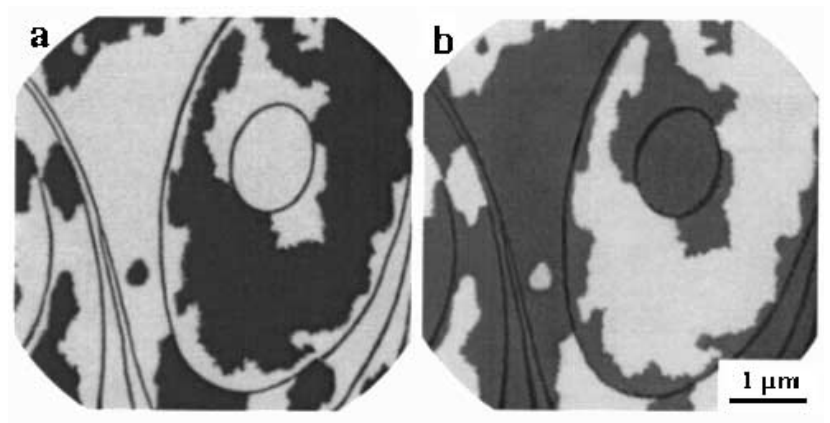

FIG. 2. LEEM images of coexisting 2 and $3 \mathrm{ML} \mathrm{Ag/W(110)}$ at imaging energy of (a) $5.8 \mathrm{eV}$ and (b) $9.8 \mathrm{eV}$. The dark lines are monatomic steps generated at the $\mathrm{Ag} / \mathrm{W}$ interface.

aged over numerous pixels, single-thickness $I(V)$ curves of the (00) beam are obtained simultaneously for all of the different thicknesses present in the image field of view. Shown in Fig. 3(a) are such $I(V)$ spectra obtained at normal incidence for $\mathrm{Ag}$ films on W(110). The first $\mathrm{Ag}$ Bragg peak is below vacuum and the second Bragg peak at about $16 \mathrm{eV}$ shifts slightly with increasing film thickness. The peaks between $0 \mathrm{eV}$ and the $\mathrm{Ag}$ Bragg peak are the prominent QIPs that come from the QSE. We use a full dynamical theory to analyze the $I(V)$ spectra. The atomic potentials of $\mathrm{Ag}$ and $\mathrm{W}$ are generated using full linear augmented plane wave [14] potentials from which phase shifts for both metals are obtained from the spherically averaged component. Details of the multiple-scattering method and inputs used are given elsewhere [12]. Note that the QIP positions, which are fit accurately, are the key features for the thickness determination. Deviation of the fit to the QIP heights below $6 \mathrm{eV}$ is attributed to the

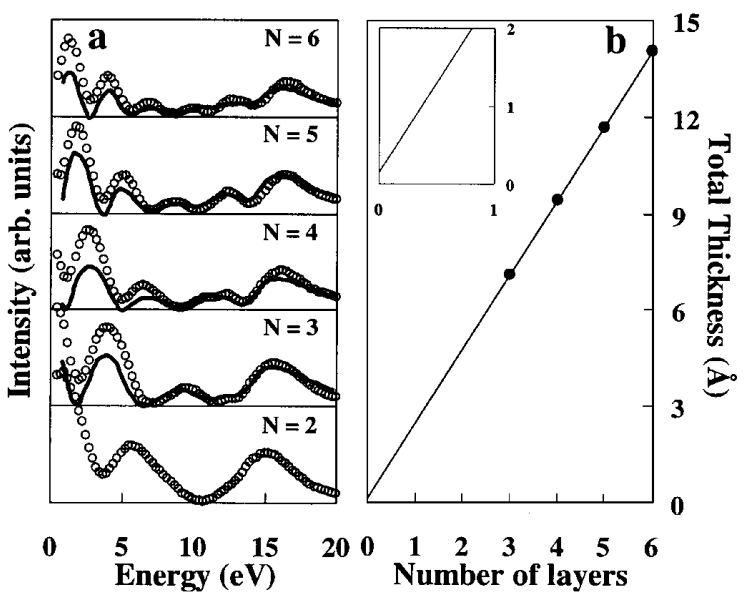

FIG. 3. (a) Quantum interference peaks due to the quantum size effect in electron reflectivity for $N=2$ to 6 layer Ag films on $\mathrm{W}(110)$ determined in experiment $(\bigcirc)$ and by dynamical theory analysis (solid curves). A Bragg peak is present at $16 \mathrm{eV}$. (b) The total thickness determined as a function of the number of atomic layers in the film. 
details of inelastic damping, which have little effect on the peak positions [12]. From the analysis [Fig. 3(a)], the total thickness of the 3, 4, 5, and 6 layer films are determined to be 7.08, 9.43, 11.68, and $14.04 \AA$ [Fig. 3(b)], respectively. These values are determined with $\pm 0.05 \AA$ uncertainties, based on the sensitive movement of theoretical peak position when the film thickness fitting parameter is varied. This sensitivity comes from the rapid variation of the wave vector at very low energy.

We find experimentally that films are below the critical thickness for the introduction of misfit dislocations in the entire thickness range studied. It is therefore worthwhile to apply the standard tools of elasticity theory [1] to solve the coherency strain problem in $\mathrm{Ag} / \mathrm{W}(110)$. An expression for the out-of-plane strain, $\tilde{\varepsilon}_{z z}$, in terms of the inplane strains can be derived [15]:

$$
\tilde{\varepsilon}_{z z}=-\frac{c_{11}+2 c_{12}-2 c_{44}}{c_{11}+2 c_{12}+4 c_{44}}\left(\tilde{\varepsilon}_{x x}+\tilde{\varepsilon}_{y y}\right),
$$

where $x, y$, and $z$ refer to the mutually orthogonal film in-

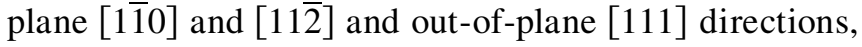
respectively, $c_{i j}(i, j=1,2,4)$ are the elastic stiffness constants, and $\tilde{\varepsilon}_{y y}=0.0313$ and $\tilde{\varepsilon}_{x x}=0$ are known from the model [10] (Fig. 1). As expected, $\tilde{\varepsilon}_{z z}$ does not depend upon the in-plane shear strain, $\tilde{\varepsilon}_{x y}=-8.72 \times$ $10^{-3} \mathrm{rad}$, which does not change the area of the twodimensional unit cell. Expressions for the shear strains, $\tilde{\varepsilon}_{y z}$ and $\tilde{\varepsilon}_{x z}$, are also determined [15]. Using values of the elastic stiffness constants from the literature [16], $c_{11}=$ $122, c_{12}=92, c_{44}=45.5$ (all in units of GPa), Eq. (1) yields a value of the out-of-plane strain, $\tilde{\varepsilon}_{z z}=-0.0136$, and shear strains, $\tilde{\varepsilon}_{y z}=-8.94 \times 10^{-3} \mathrm{rad}$ and $\tilde{\varepsilon}_{x z}=$ $-8.09 \times 10^{-3} \mathrm{rad}$. The perpendicular lattice constant of the strained film, $a_{\perp}$, is related to the bulk $\operatorname{Ag}(111)$ layer spacing, $a_{\perp 0}=2.361 \AA$, as $a_{\perp}=a_{\perp 0}\left(1+\tilde{\varepsilon}_{z z}\right)$, which yields $a_{\perp}=2.329 \AA$.

The Ag on W total energy calculations were carried out by using local-density-functional formalism, employing a plane-wave basis and ultrasoft pseudopotentials $[17,18]$. The cutoff kinetic energy of the plane-wave basis was set at $17.30 \mathrm{Ry}$. We use the standard repeated slab geometry, which consists of 19 atomic layers and a vacuum of $12 \AA$. All atomic positions were relaxed until the magnitude of the Hellmann-Feynman forces became less than $0.1 \mathrm{eV} / \AA$, and the total energy converged to the order of $1 \mathrm{meV}$. A $12 \times 12$ uniform grid in the two-dimensional Brillouin zone was used for $k$-point sampling [19].

First-principles calculations of strained $\mathrm{Ag}(111)$ films on the $\mathrm{W}(110)$ surface are prohibited by the incommensurate relationship in the substrate [1i1] direction. Instead, we have calculated layer spacings for five different related systems and offer convincing arguments that justify comparison of these results to the experimental system. The five systems under consideration in the calculation are as follows: (I) freestanding fcc $\mathrm{Ag}(111)$ layers; (II) freestanding strained fcc $\operatorname{Ag}(111)$ layers; (III) strained fcc $\operatorname{Ag}(111)$ layers on W adopting the same lateral strained $\mathrm{fcc}(111)$ structure (strained $\mathrm{Ag} / \mathrm{W}$ ); (IV) freestanding Ag films with bcc W(110) structure and in-plane lattice constant; (V) pseudomorphic Ag films on bcc W(110) (ps Ag/W). The lateral strain in models II and III follows Fig. 1(b). In all cases, 19 layer slabs are considered. The $\mathrm{Ag} / \mathrm{W}$ configurations (models III and V) are generated by replacing the interior seven layers of the related freestanding $\mathrm{Ag}$ layer systems (models II and IV, respectively) with W atoms.

The results for the layer spacings in six layer Ag films on $\mathrm{W}$ and the top six layer spacings in the case of the freestanding $\mathrm{Ag}$ layers are shown in Fig. 4(a). In this figure, the spacing between the surface and the first subsurface Ag layer is identified as "surface" and the spacing between the bottom Ag layer and top W layer of the substrate is labeled "interface." The first comparison to make is between (I) freestanding $\operatorname{Ag}(111)$ and (II) freestanding strained $\operatorname{Ag}(111)$ layers. The reduction of the interlayer spacing in the strained layers is in quantitative agreement with elasticity theory predictions. These results also show that the surface relaxation, which is confined to the two topmost layer spacings, is influenced by lateral strain in the same way as the deeper bulklike layers. The calculated surface relaxation is in agreement with prior density functional theory results [20] and with experimentally determined values for $\operatorname{Ag}(111)$, although the previous experimental works are slightly at odds over how the relaxation is distributed in the top two layers [21,22].

Comparing the strained $\mathrm{Ag} / \mathrm{W}$ systems (models II and III), we see that the influence of $\mathrm{W}$ is localized at the $\mathrm{Ag}$ $\mathrm{W}$ interface layer. Layer spacings in the $\mathrm{Ag}$ film beyond the interface layer are unaffected by the introduction of $\mathrm{W}$ at the interior of the slab. For $\mathrm{ps} \mathrm{Ag} / \mathrm{W}$ (compare models IV and V), however, the first two Ag layer spacings are modified strongly by the presence of an interface

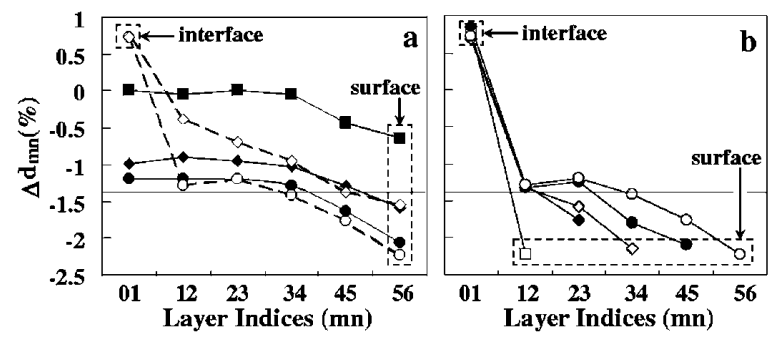

FIG. 4. First-principles results for spacings between layers $m$ and $n$ relative to bulk $\operatorname{Ag}(111)$ (a) in (I) freestanding $\operatorname{Ag}(111)$ layers (ם), (II) freestanding strained $\operatorname{Ag}(111)$ layers (O), (III) strained $\mathrm{Ag} / \mathrm{W}(\mathrm{O})$, (IV) freestanding bcc $\mathrm{Ag}(110)$ layers $(\diamond)$, and $(\mathrm{V}) \mathrm{ps} \mathrm{Ag} / \mathrm{W}(\diamond)$ films, and (b) in strained $\mathrm{Ag} / \mathrm{W}$ (model III) for different numbers of Ag layers, $N=2(\square), 3$ $(\diamond), 4(\diamond), 5(\diamond), 6(\bigcirc)$. The horizontal line indicates the elasticity theory prediction for model II (see text). 
with a W substrate. Therefore, we attribute the very strong screening of the $\mathrm{W}$ by just one $\mathrm{Ag}$ layer in strained $\mathrm{Ag} / \mathrm{W}$ to the $\mathrm{Ag}$ fcc stacking. This strong screening of the substrate justifies the use of the strained $\mathrm{Ag} / \mathrm{W}$ (model III) results as a model for the strained Ag films studied in the experiment.

We next turn our attention to the dependence of layer spacings upon the number of $\mathrm{Ag}$ layers for strained $\mathrm{Ag} / \mathrm{W}$ [Fig. 4(b)]. Interestingly, the interface layer spacing is independent of the number of atomic layers in the film, and layers spacings in the interior of the film are close to the value predicted by elasticity theory. The surface relaxation of the film is also largely independent of the number of layers. A remarkably simple model of layer spacings in coherently strained fcc(111) films emerges from the first-principles calculations. In this model, strained bulk layer spacings (including similarly modified surface relaxations) are present up to the layer in contact with the substrate. If an atomic layer is added to the film, then the total film thickness increases by the strained bulk layer spacing. This quantity may be determined by measuring the incremental change of the total film thickness each time the number of layers in the film is increased by one. This corresponds to the slope of the line in Fig. 3(b). Adopting this model for the $\mathrm{Ag} / \mathrm{W}(110)$ system under investigation, we determine the strained layer spacing at the interior of the film to be $a_{\perp}=2.32 \pm$ $0.05 \AA$. This is in good agreement with first-principles calculations and elasticity theory, although still marginally within uncertainty of the unstrained bulk spacing. After taking the surface relaxation into account, the interface layer spacing may be determined from the $y$ intercept in Fig. 3(b) (inset) when the total film thickness is extrapolated to zero layers. The average interface layer spacing is determined to be $a_{\text {int }}=2.47 \pm 0.06 \AA$. Thus, the experiment is in qualitative agreement with firstprinciples calculations that the interface layer spacing exceeds both the Ag film and the W substrate layer spacings. It is important to note that the calculation employs a $\mathrm{W}$ two-dimensional lattice that is slightly expanded from the $\mathrm{W}(110)$. This reduces the electron density at the interface and leads to an underestimate of the interface layer spacing by the calculation. Taking this into account, the calculation and experiment are in reasonably good agreement.

In conclusion, we have exploited the QSE in electron reflectivity to determine the total film thickness of coherently strained $\operatorname{Ag}(111)$ films on $\mathrm{W}(110)$. Laterally resolved measurements of the QSE in a low energy electron microscope are crucial for determining how the total film thickness evolves with increasing number of atomic layers. This information is the basis for a novel approach to evaluate the strained layer and buried interface layer spacing, the latter of which is inaccessible to other methods. The experimentally determined strained layer spacing is in good agreement with first-principles calculations and elasticity theory. The interface layer spacing is also found experimentally to be expanded relative to both $\mathrm{Ag}(111)$ and $\mathrm{W}(110)$ layer spacings, which is in qualitative agreement with first-principles calculations but cannot be addressed by elasticity theory. This work also points the way to wider application of this and other QSEs for evaluating details of film structure.

This work was supported by the Hong Kong Research Grants Council under Grants No. HKUST6129/98P, No. HKUST6152/01P, and No. CityU1238/02P.

*Corresponding author.

Email address: phaltman@ust.hk

[1] J. F. Nye, Physical Properties of Crystals (Oxford University Press, Oxford, 1957).

[2] E. Chason and T. M. Meyer, Crit. Rev. Solid State Mater. Sci. 22, 1 (1997), and references therein.

[3] B. T. Jonker, N. C. Bartelt, and R. L. Park, Surf. Sci. 127, 183 (1983).

[4] D. Kerkmann, D. Pescia, J.W. Krewer, and E. Vescovo, Z. Phys. B 85, 311 (1991).

[5] E. Bauer, Rep. Prog. Phys. 57, 895 (1994).

[6] M. S. Altman, W. F. Chung, and C. H. Liu, Surf. Rev. Lett. 5, 1129 (1998).

[7] T. C. Chiang, Surf. Sci. Rep. 39, 181 (2000).

[8] D. Chen, J. Electron Spectrosc. Relat. Phenom. 109, 85 (2000).

[9] L. Abelle, C. Rogero, and K. Horn, Phys. Rev. B 65, 125319 (2002).

[10] E. Bauer et al., J. Appl. Phys. 48, 3773 (1977).

[11] Y.W. Yang, H. Xu, and T. Engel, Surf. Sci. 276, 341 (1992).

[12] H. C. Poon et al., Surf. Rev. Lett. 5, 1143 (1998).

[13] V. Yeh et al., Phys. Rev. Lett. 85, 5158 (2000).

[14] H. J. F. Jansen and A. J. Freeman, Phys. Rev. B 30, 561 (1984).

[15] B. Foreman and M. S. Altman (unpublished).

[16] R. F. S. Hearmon, in Elastic, Piezoelectric, Pyroelectric, Piezooptic, Electrooptic Constants, and Nonlinear Dielectric Susceptibilities of Crystals, edited by K.-H. Hellwege and A. M. Hellwege, Landolt-Börnstein, New Series, Group III, Vol. 18 (Springer, Berlin, 1984), p. 4.

[17] D. Vanderbilt, Phys. Rev. B 41, 7892 (1990); G. Kresse and J. Hafner, J. Phys. Condens. Matter 6, 8245 (1994).

[18] G. Kresse and J. Hafner, Phys. Rev. B 47, R558 (1993); G. Kresse and J. Furthmüller, Phys. Rev. B 54, 11169 (1996).

[19] H. J. Monkhorst and J. D. Pack, Phys. Rev. B 13, 5188 (1976).

[20] Jianjun Xie et al., Phys. Rev. B 59, 970 (1999).

[21] P. Statiris, H. C. Lu, and T. Gustafsson, Phys. Rev. Lett. 72, 3574 (1994).

[22] E.S. Soares et al., Surf. Sci. 468, 129 (2000). 